\title{
Coordinates and 2MASS and OGLE identifications for all stars in Arp's 1965 finding chart for Baade's Window ${ }^{\star}$ (Research Note)
}

\author{
R. P. Church ${ }^{1}$, J. A. Johnson ${ }^{2,1, \star \star}$, and S. Feltzing ${ }^{1}$ \\ 1 Lund Observatory, Department of Astronomy and Theoretical Physics, Box 43, 22100 Lund, Sweden \\ e-mail: [ross; sofia]@astro.lu.se \\ 2 Department of Astronomy, Ohio State University, 140 W. 18th Avenue, Columbus, OH 43210, USA \\ e-mail: jaj@astronomy.ohio-state.edu
}

Received 4 March 2011 / Accepted 23 March 2011

\section{ABSTRACT}

\begin{abstract}
Aims. We seek to provide 2MASS and OGLE identifications and coordinates for all stars in the finding chart published by Arp (1965, ApJ, 141, 43). This chart covers the low extinction area around NGC 6522, also known as Baade's window, at coordinates $(l, b)=$ $(1.02,-3.92)$.

Methods. A cross correlation, using numerical techniques, was performed between a scan of the original finding chart from Arp and 2MASS and OGLE-II images and stellar coordinates.

Results. We provide coordinates for all stars in Arp's finding chart and 2MASS and OGLE identifications wherever possible. Two identifications in quadrant II do not appear in the original finding chart.
\end{abstract}

Key words. catalogs - Galaxy: bulge - globular clusters: individual: NGC 6522

\section{Introduction}

The region towards the Galactic Bulge situated at Galactic coordinates $(l, b)=(1,-4)$ known as Baade's window is a much studied area as it provides a low extinction region where we are able to look deeper into the Galactic Bulge than in most other regions. This region is centred on a globular cluster, NGC 6522. Halton Arp (1965) provided a much-used finding chart for the immediate region around the cluster. This article also provided broad band photometry for all the labelled stars. This finding chart has proved important especially for the identification of stars suitable for high resolution spectroscopy (e.g., McWilliam \& Rich 1994; Fulbright et al. 2006; Ryde et al. 2009). However, Arp (1965) did not publish coordinates for the stars, and very few of the spectroscopic studies using his finding chart have done so. Indeed, only 37 of the stars in Arp (1965) have SIMBAD entries (according to ADS 2011-02-15).

In the era of large surveys, such as Sloan Digital Sky Survey (SDSS) and The Two Micron All Sky Survey (2MASS, Skrutskie et al. 2006), old numbering schemes for relatively small patches of the sky may appear of less relevance. However, for correct comparison with the high resolution studies it is important that we know which star has which identification number in Arp's catalogue and which 2MASS and Optical Gravitational Lensing Experiment (OGLE) identifications it has. We suspect that others have carried out the same painful exercise as we have done a few times and truly cross-identified some of, e.g., the Fulbright et al. (2006) stars with their correct 2MASS identification. We are, however, not aware of any such list available.

\footnotetext{
* Table 1 is only available in electronic form at the CDS via anonymous ftp to cdsarc.u-strasbg.fr (130.79.128.5) or via http://cdsarc.u-strasbg.fr/viz-bin/qcat?J/A+A/529/A104 $\star \star \mathrm{J}$. A. Johnson is a guest professor at Lund Observatory.
}

We therefore decided to provide a complete cross identification between the Arp numbers and 2MASS and OGLE identifications and provide coordinates for all stars on the finding chart in Arp (1965).

\section{Method of cross identification}

To obtain the coordinates of the stars in Arp's sample, we took a scan of figure 1 of Arp (1965) at 600 dpi using an office photocopier. The halftone pattern produced by the printing process was removed by iteratively applying a simple algorithm in which white pixels with predominantly black neighbours were coloured black and vice-versa. Having done this we identified all contiguous regions of black points and analysed each in turn, in an attempt to determine whether they were stars or Arp's annotations. The smallest regions ( $<50$ pixels) were disregarded, and regions with a high aspect ratio were marked as lines. Similarly, thin sections of regions were marked as lines protruding from stellar images. The remaining regions contained predominantly stars and numeric digits. We selected stars as regions that had relatively high surface density; i.e. where $>60 \%$ of the rectangular area encompassing the region was black pixels. We found this to work well as stars tended to be solid blocks of pixels, whereas numbers tend to contain empty regions. Empirically we also found it useful to repeat this test with the outer $20 \%$ of the region removed and using a higher threshold of $66 \%$. Unusually large regions ( $>750$ pixels) were marked as stars, as well as small regions $(<300$ pixels, but not including the very small regions disregarded above). Whilst a more sophisticated probabilistic model might have been more accurate, these empirical rules served to separate stars from annotation marks with an accuracy of about $90 \%$. 
For each identified stellar image we calculated the centroid. We checked the location of the centroid of each identified star by eye and adjusted those where the algorithm had performed badly. In many cases a pair of stellar sources had become blended into a single region; in these cases the centroids of the two stars were extracted using the K-means++ algorithm (Arthur \& Vassilvitskii 2007). We then matched each stellar object with its number - if any - in Arp's catalogue by hand.

In order to cross-identify Arp's sources with 2MASS sources we used the sample of Blanco (1986) and positions from Glass $\&$ Schultheis (2002) to produce an initial mapping between pixel coordinates in our scanned image and sky positions. Right ascension and declination were fit as linear combinations of $x$ and $y$ in pixel space, with a separate fit being made for each of Arp's quadrants. We used this fit to identify, for each star, the closest 2MASS source. Where the closest source was within 2 arcseconds, this was taken to be a tentative match, and the fit repeated using the catalogued positions of these stars. We checked each match by eye to see whether it appeared to be genuine; in some cases a single 2MASS source was seen to be a blending of two sources in Arp's catalogue. Some of Arp's stars were found to have no corresponding source in 2MASS. For these sources, and sources which appeared to be blended into one 2MASS source we present coordinates derived from our fitting; otherwise the coordinates presented are those of the corresponding 2MASS source. That not all stars in Arp's finding chart have 2MASS counterparts is a natural consequence of the redder filters used in 2MASS and the fact that the two surveys reach different depths.

Finally we repeated the fitting process to match stars in the OGLE catalogue, where the cross-over was more complete. All OGLE sources identifications are in the BUL SC45 field and we used an $I$-band template image from OGLE-II for the crosscorrelation (Udalski et al. 2002).

Identification numbers II-7 and 39 appear to not have any counterparts on the original finding chart in Arp (1965).

\section{Table of coordinates, identifiers, and photometry}

Table 1 presents our results for Arp's quadrants I, II, III and IV. Here we give a brief description of the contents of Table 1.

Id number: the first two columns give the identifications from the finding chart in Arp (1965). The first column gives them as Arabic digits; the first digit indicates the quadrant. The second column gives the classical notation used in Arp (1965) with quadrants indicated by Roman numerals.

Coordinates: coordinates are from 2MASS whenever available, for the remaining stars we give the coordinates as calculated in Sect. 2. The coordinates are in J2000 and are on the system set by 2 MASS.

2MASS (incl. blends) and OGLE identifications: we list the 2MASS and OGLE identifications and for 2MASS we also indicate whether or not the identification was a blend (see Sect. 2).

Photometry: for convenience we include the photometry from 2MASS $\left(\mathrm{JHK}_{\mathrm{S}}\right)$ and OGLE-II (VI photometry Udalski et al. 2002) in the table. We have included certain flags regarding the quality of the 2MASS photometry. The description of the meaning of the relevant flags are given in Appendix A. We include the errors in the photometry for both 2MASS and OGLE data.

\section{Summary}

We present coordinates for all stars identified in the finding chart by Arp (1965) of the globular clusters NGC 6522, also known as Baade's window. This is a much studied low extinction area situated close to $(l, b)=(1,-4)$. The coordinates are on the 2 MASS system.

As part of the process we have produced, in a semiautomated fashion, a match between the sources in Arp (1965) and the 2MASS and OGLE catalogues.

Acknowledgements. R.P.C. is funded by a Marie-Curie Intra-European Fellowship, grant No. 252431, under the European Commission's FP7 framework. J.A.J. acknowledges a guest professorship provided by the Faculty of Science, Lund University. S.F. was partly supported by grant No. 2008-4095 from The Swedish Research Council.

This research has made use of the SIMBAD database, operated at CDS, Strasbourg, France.

This publication makes use of data products from the Two Micron All Sky Survey, which is a joint project of the University of Massachusetts and the Infrared Processing and Analysis Center/California Institute of Technology, funded by the National Aeronautics and Space Administration and the National Science Foundation.

\section{Appendix A: Flags for 2MASS photometry}

$\mathrm{A}=$ Detections in any brightness regime where valid measurements were made with [jhk]snr $>10$ AND [jhk]cmsig $<0.10857$. $\mathrm{B}=$ Detections in any brightness regime where valid measurements were made with [jhk]snr $>7$ AND [jhk]cmsig $<0.15510$. $\mathrm{C}=$ Detections in any brightness regime where valid measurements were made with [jhk] snr $>5$ AND [jhk]cmsig $<0.21714$. $D=$ Detections in any brightness regime where valid measurements were made with no [jhk]snr OR [jhk]cmsig requirement.

$E=$ This category includes detections where the goodness-of-fit quality of the profile-fit photometry was very poor, or detections where psf fit photometry did not converge and an aperture magnitude is reported, or detections where the number of frames was too small in relation to the number of frames in which a detection was geometrically possible.

$U=$ Upper limit on magnitude. Source is not detected in this band, or it is detected, but not resolved in a consistent fashion with other bands.

\section{References}

Arp, H. 1965, ApJ, 141, 43

Arthur, D., \& Vassilvitskii, S. 2007, in Proceedings of the eighteenth annual ACM-SIAM symposium on Discrete algorithms, SODA '07 (Philadelphia, PA, USA: Society for Industrial and Applied Mathematics), 1027 Blanco, V. M. 1986, AJ, 91, 290

Fulbright, J. P., McWilliam, A., \& Rich, R. M. 2006, ApJ, 636, 821 Glass, I. S., \& Schultheis, M. 2002, MNRAS, 337, 519 McWilliam, A., \& Rich, R. M. 1994, ApJS, 91, 749

Ryde, N., Edvardsson, B., Gustafsson, B., et al. 2009, A\&A, 496, 701 Skrutskie, M. F., Cutri, R. M., Stiening, R., et al. 2006, AJ, 131, 1163 Udalski, A., Szymanski, M., Kubiak, M., et al. 2002, Acta Astron., 52, 217 